\title{
Polymorphism and solvates. Application to pharmaceutical compounds
}

\section{B. Legendre}

\section{Laboratoire de Chimie-Physique Minérale et Bioinorganique, EA 401, Faculté de Pharmacie, Rue J.B. Clément, 92290 Châtenay-Malabry, France}

\begin{abstract}
This article is the text of the lecture presented for the XXVII JEEP in Montpellier.
Polymorphism is well known in inorganic chemistry, it is relative to the variation of temperature and pressure. In organic chemistry the subject is more delicate because it does not depend only on these two functions, but also the way of preparation of the substance, the nature of the solvent of crystallisation, its rate of evaporation, and for melted compounds, the rate of cooling is also an important factor. In these conditions the study of polymorphism cannot be dissociated of the study of solvates. General knowledge will be presented in this paper, but also some particular cases in which equilibrium is difficult to reach.
\end{abstract}

\section{INTRODUCTION}

The oldest description of a polymorphism case is related by Klaproth in 1788 , about the calcite $\mathrm{CaCO}_{3}$, in 1796 Tennant discovered that a diamond is just a form of carbide, it was hard for these scientists to make themselves believed. The term allotropy is reserved for the elements and polymorphism for molecular compounds. The most acceptable definition for the polymorphism is: "the possibility for a compound to exist in the solid crystallised state within different forms". Each form has its own physical properties, and it is the reason why a study of polymorphism is of paramount importance. To approach the problems of polymorphism, two basic sciences are necessary : crystallography and thermodynamics. We shall describe in this article the main concepts necessary to resolve the problems, and which techniques must be developed. Some examples will be exposed, especially, some cases presenting difficulties of interpretation, they correspond to cases for which equilibrium is difficult to reach, but this will be explained by the exploitation of the Gibbs function $G=f(T)$ and the free energy of activation used in thermokinetics.

\section{THERMODYNAMIC APPROACH}

According to Gibbs's rule only one form is thermodynamically stable for one pressure and one temperature, the others are metastable

$$
\mathrm{V}=\mathrm{n}+\mathrm{P}-\varphi
$$

$\mathrm{V}$ is the variance; in fact it represents the number of degrees of freedom, $\mathrm{n}$ is the number of independent constituents, $\mathrm{P}$ is the number of intensive variable $(\mathrm{P}, \mathrm{T} \ldots), \varphi$ is the number of phases, for one component, if $\mathrm{P}$ and $\mathrm{T}$ are fixed $\varphi$ must be equal to 1 , if two solid phases exist, only one will be in a stable state from a thermodynamic point of view.

For two polymorphs, the melting points and the enthalpies of fusion are different, this is of a prime importance, as we shall see in this article, the rate of dissolution depends of the enthalpy of fusion, consequently the bio-availability depends on the heat of fusion and that the study of the polymorphism is of paramount importance for pharmacokinetics. We shall present in this article different examples of polymorphism, and especially some rather complicated cases in which thermodynamic behaviour can be explained with the help of the kinetics.

A nice diagram is always more pleasant than a differential equation, in view to understanding the polymorphism we shall use P, $\mathrm{T}$ unary diagram, $\Delta H=\mathrm{f}(\mathrm{T})_{\mathrm{p}}, G=\mathrm{f}(\mathrm{T})_{\mathrm{p}}$. The first question is : is it possible to predict the polymorphism?" I would say yes in some cases and I will explain why. 
The most well known unary PT diagram is that of water represented in figure 1a. As we may observe the slope of the bounding curve between the solid and the liquid state which

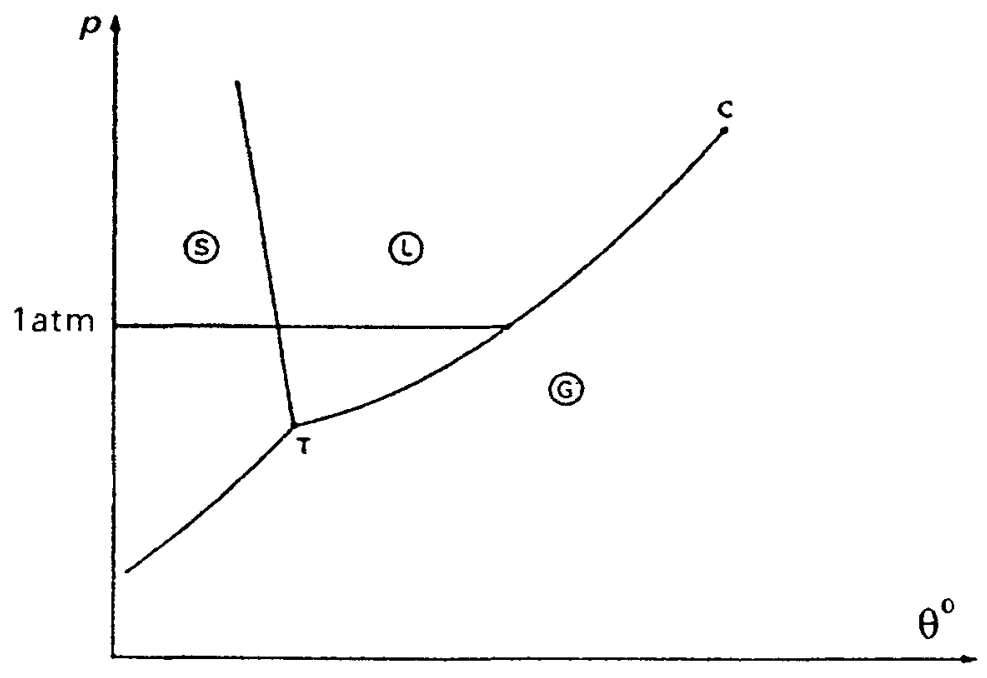

Figure 1a: $\mathrm{P}, \mathrm{T}$ diagram of water

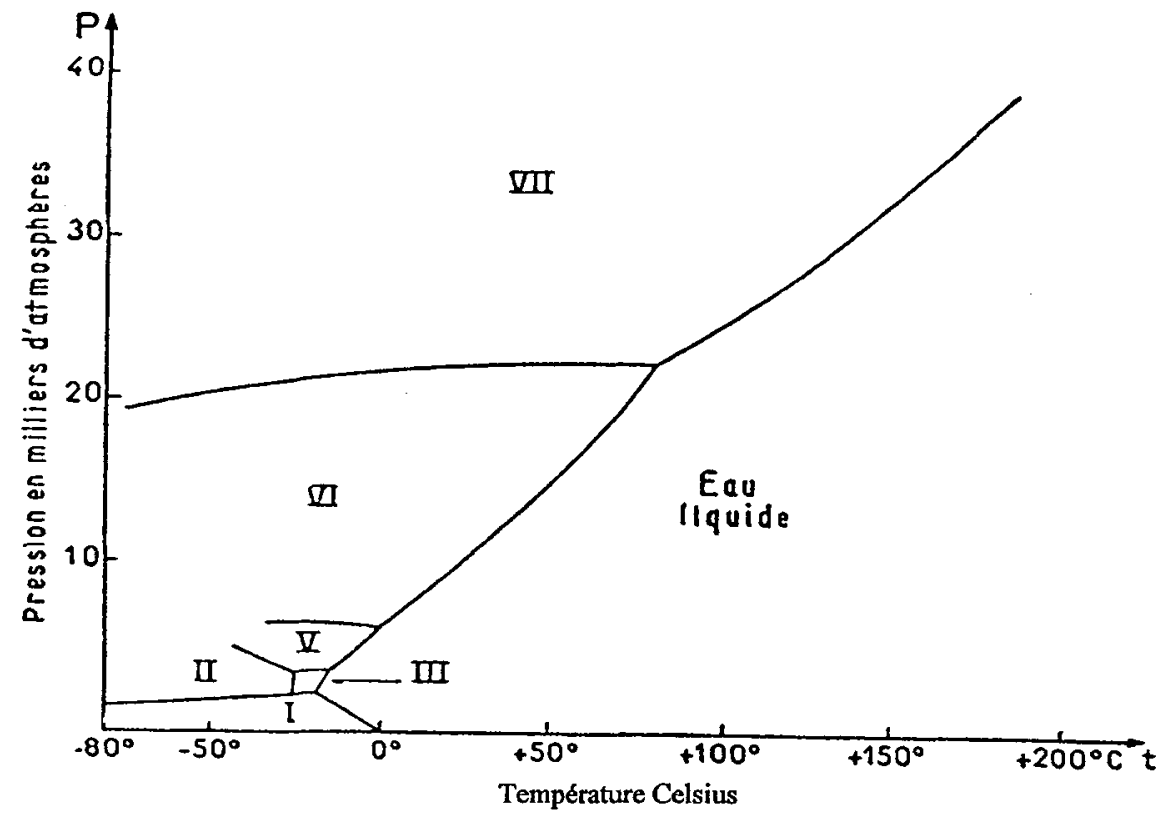

Figure $1 \mathrm{~b}: \mathrm{P}, \mathrm{T}$ diagram of water at high pressure 
represent the fusion $\mathrm{dP} / \mathrm{dT}$ is negative. Let us consider a point in the gas state, if the temperature is kept constant, and if the pressure increases, we will observe a phase transformation, it will be the liquefaction of the gas, it means that if the molecules are closer together, the system will be transformed from an expensed system to a condensed system, according to this argument, if the pressure increases in the liquid state the system will reach a more condensed state, and this state must be the solid state. Apparently it seems impossible with a negative slope $P=f(T)$. Of course we may have a doubt about the sign of this slope, but using the relation of Clapeyron : $\mathrm{dP} / \mathrm{dT}=\left(\Delta_{\mathrm{ftus}} H / \mathrm{T} \Delta \mathrm{V}\right), \Delta \mathrm{V}$ is the difference between the volume of the liquid and the volume of the solid, as the enthalpy of fusion and $\mathrm{T}$ are positive, $\Delta \mathrm{V}$ must be negative, and as everyone knows, the volume of ice is greater than the volume of liquid water. If the unary phase diagram is studied at high pressure ( $P>2000$ bars), polymorphic forms appears figure $1 \mathrm{~b}$. It has been observed for a good number of unary diagrams, that each time that $\mathrm{dP} / \mathrm{d} \mathrm{T}$ is negative, there is at least one high pressure phase. This can be accepted as a general rule, but cannot be demonstrated.

It is possible to measure the melting temperature of a compound at different pressures and to deduce the slope of the fusion curve, these kinds of measurements are not used routinely because they require sophisticated apparatus. Most of the time the polymorphism is discovered by chance, as crystallisation of the produce in different solvents and observation of the X-ray pattern, or by. DSC (different scanning calorimetry). But if we want to know if polymorphism exists, the best way is the establishment of the unary phase diagram $(P, T)$.

For organic compounds it is possible to obtain high-pressure phases by increasing the pressure, but also by processes of dissolution in various solvents and crystallisation by evaporation of the solvent.

We can explain this phenomenon by analogy with the surrounded atom model of Mathieu [1] and Lupis [2], this model is developed for metallic solutions. For organic compounds in solution, generally the molecules of the solvent are of a smaller size than the molecules of the solute. When a solid is dissolved in the solvent, the number of moles of solvent is more important that the number of mole of solute, and one molecule of solute is in fact surrounded by a great number of molecules of solvent as first and second neighbours, and solute molecules are submitted to a field induced by the molecules of solvent, the field depends of the electric dipoles, and will not be the same for a polar and a non polar molecule, but also the geometry of the molecule of solvent has an influence on the shape of the molecules of solute in solution. This is well known for solution of water and alcohol, the volume of the solution composed with $\mathrm{H}_{2} \mathrm{O}$ and $\mathrm{C}_{2} \mathrm{H}_{5} \mathrm{OH}$ is not the sum of the volume of each constituent, the partial molar properties for alcohol depend of the nature of the other compound, its composition and the temperature. In fact we may consider that the molecules of solvent act as a stamping press and if different solvents are used, it is the same thing that if different awls are used to stamp a metallic piece, the shape of the metallic piece will not be the same. When the solvent evaporates the molecules of the solution will split in solid and liquid, the solid part will crystallise, with the same shape that in the liquid, but sometime the relaxation leading to the most stable configuration is very fast, it can last a fraction of second or a few hours. At that stage analysis must be performed in view to verify that the solid is not a solvate, TGA and X-ray will be the right tools for this verification. We shall come back later in this article to the problem of germination and solid-solid reaction.

The obtaining of different crystallographic forms has been observed in a lot of cases, but following the phase rule, only one form can be in a thermodynamic stable state, then it is possible to observe' in the time an evolution of the metastable phase transforming in the stable 
phase, the kinetic of a solid-solid reaction is always slow, it may last a few hours or some months.

Different polymorphs of a drug may exhibit significantly different biological activities due to their different solubility and dissolution rate[3].

The rate of dissolution depends on the enthalpy of dissolution, which in fact is the sum of three terms in accordance with the first law of thermodynamics:

$$
\Delta_{\text {diss }} \boldsymbol{H}=\int_{\mathrm{TO}}^{\mathrm{Tfus}}\left(\mathrm{Cp}^{\mathrm{s}}-\mathrm{Cp}^{\mathrm{l}}\right) \mathrm{dT}+\Delta_{\text {fus }} \boldsymbol{H}+\Delta_{\text {mix }} \boldsymbol{H}
$$

(details of the thermodynamic cycle are presented in annex)

The first term of the second part of this equation is generally slightly negative and is close to zero. The second term is the heat of fusion, this is always positive and differs for each crystallographic form. The third term $\Delta_{\text {mix }} H$ is the enthalpy of the mixing of the solvent and of the compound in an undercooled liquid state, which may be positive or negative, but depends only on the liquid state, and is independent of the crystallographic form.

Experimentally it is possible to measure the enthalpy of dissolution, the $\mathrm{Cp}$ of the solid phase and the $\mathrm{Cp}$ of the liquid in a range of temperature close to the melting point, for organic compounds, the enthalpy of mixing is only deduced, a direct measurement is impossible in most cases. As we can see, the main term for the modification of the enthalpy of dissolution is the enthalpy of fusion, we can easily deduce that bio-availability depends on the heat of fusion and that the study of the polymorphism is of paramount importance for pharmacokinetics. We shall see later how to measure these parameters.

\section{UNARY P-T PHASE DIAGRAM}

\subsection{Enantiotropic case}

Enantiotropy means that a reversibility exists when the product is heated over the melting temperature, if the cooling is performed with conditions of equilibrium the same phase will be observed. A well-known diagram is sulphur (figure 2), the plan is divided in four parts, corresponding to the area of stability, $\alpha$ and $\beta$ are the two solids forms $L$ and $G$ corresponds respectively to the liquid and the gas form. For a pressure of one atmosphere, if the sample is heated slowly, it means in conditions of equilibrium, and these conditions are not the same for all products, the solid- solid $\alpha / \beta$ transition will be observed. This transition is of the first order, according to the classification of Erhenfest, the temperature is constant during the transition, and comes with an enthalpy of transition which is always endothermic for heating, and exothermic for cooling. But the rate of a solid- solid transition is always weak, if the rate of heating is too fast the reaction does not occur, and the $\alpha$ phase is after the temperature of transition in a metastable state and this phase will melt when the temperature reaches the line joining the triple point which is inside the triangle, and the top of this triangle corresponds to the limit of the existence of the $\beta$ phase, this dashed line corresponds to the metastable fusion 


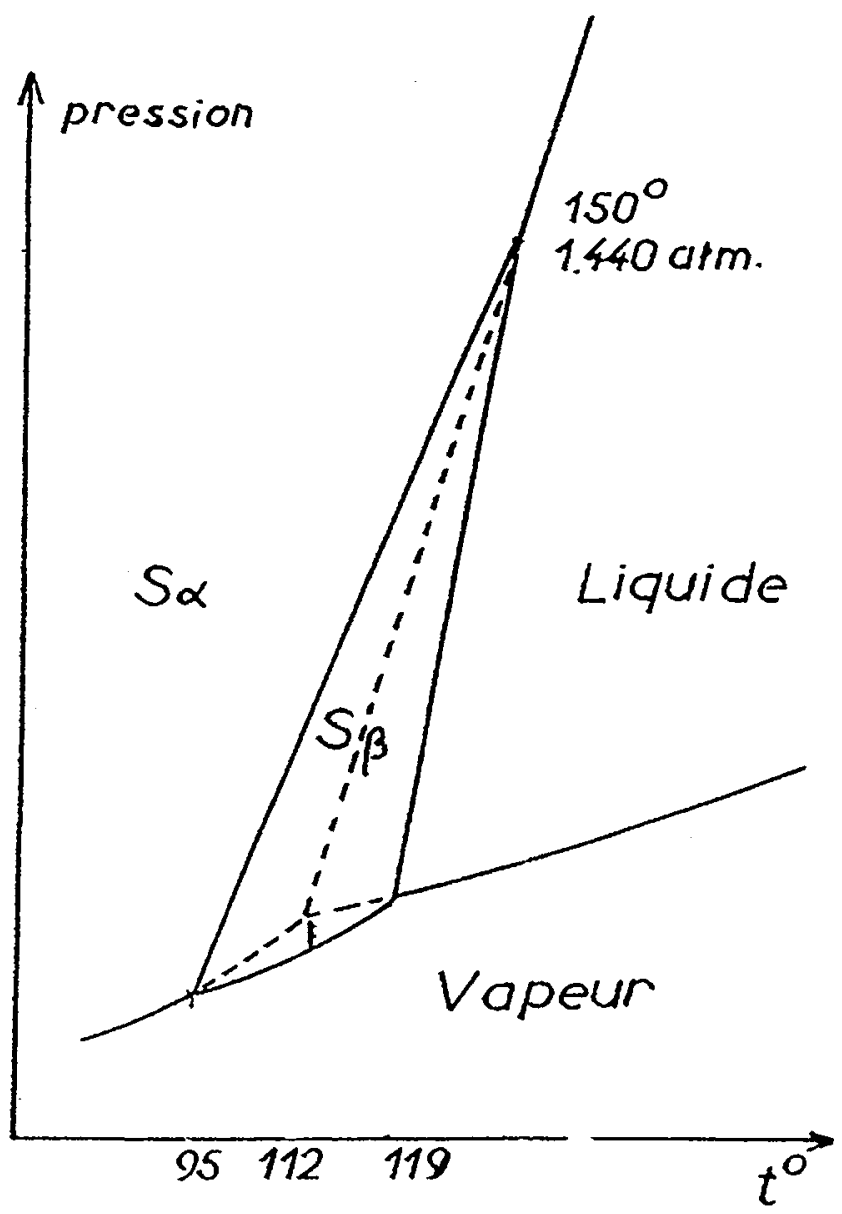

Figure $2: P, T$ Unary phase diagram of an enantiotropic case

of the $\alpha$ phase. The heat content between room temperature and $\mathrm{T}$ is given on the figure 3 . The enthalpy of fusion of the metastable phase is always higher that the enthalpy of fusion of the stable phase at its temperature of fusion. This remark is of importance, because if the temperature of transition between the solid phases is at low temperature the transition is not observed by DSC, and in this case, it is not obvious whether it is an enantiotropic case or not. If the solid phase which has the highest melting temperature presents an enthalpy of fusion higher that the one which presents a lower melting temperature, it will not be an enantiotropic case. 


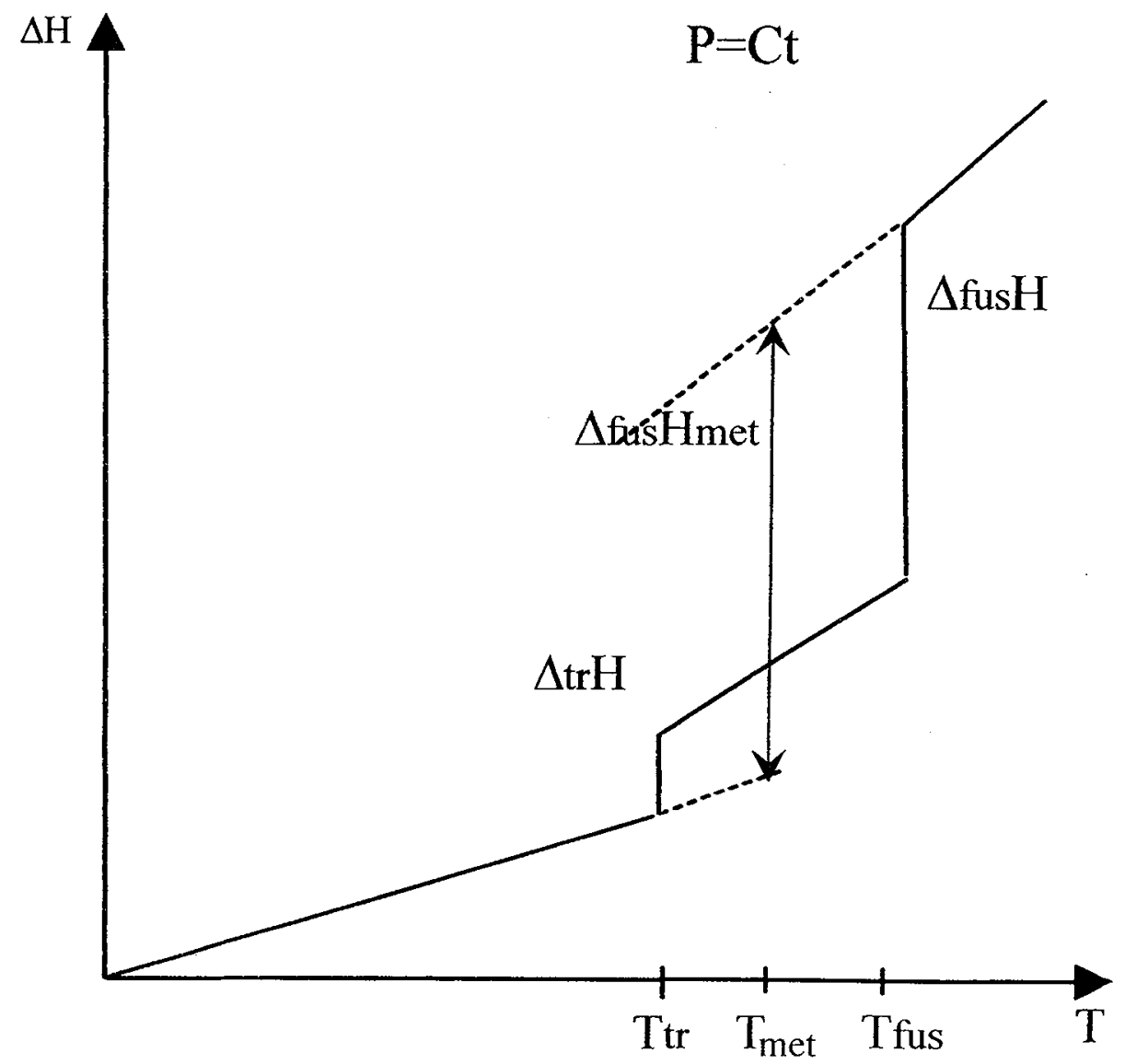

Figure 3 : Heat content versus of $\mathrm{T}, \Delta^{\mathrm{T}}{ }_{298} H=\mathrm{f}(\mathrm{T})$

The justification of the thermodynamic stability is given by the curve $G=\mathrm{f}(\mathrm{T})$ at constant pressure.

If $\mathbf{P}$ is constant :

$$
\begin{gathered}
d G=-S d T \\
\left(\frac{\partial G}{\partial T}\right)_{p}=-S
\end{gathered}
$$

The slope will be negative as its bend because the second derivative of $\mathrm{G}$ is negative : 


$$
\left(\frac{\partial^{2} G}{\partial T^{2}}\right)_{p}=-\left(\frac{\partial S}{\partial T}\right)_{p}=-\frac{C_{p}}{T}
$$

$\mathrm{Cp}$ is always positive and : $-\frac{\mathrm{C}_{\mathrm{p}}}{\mathrm{T}}$ will be negative, in consequence the curve $\mathrm{G}=\mathrm{f}(\mathrm{T})$ is always concave (figure 4 ).

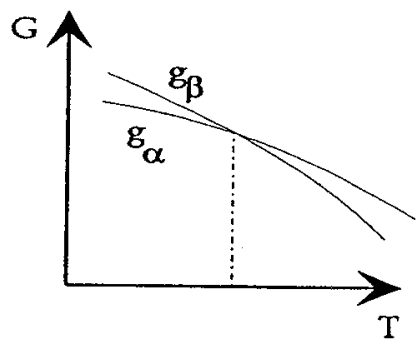

Figure 4 : Free enthalpy versus of $\mathrm{T}, G=\mathrm{f}(\mathrm{T})$

The most stable phase for one temperature and a fixed pressure, is the one for which the Gibbs energy $\mathrm{G}$ is the lowest.

The study of carbamazepine is an interesting enantiotropic case, it has been studied in our laboratory. Different scanning calorimetry has been performed with a Perkin - Elmer (DSC 7) using a rate of $10 \mathrm{~K} / \mathrm{min}$, a small endothermic peak of $9 \mathrm{~J} / \mathrm{g}$ was observed at $155^{\circ} \mathrm{C}$, and another peak of greatest intensity at $189.9^{\circ} \mathrm{C}(110 \mathrm{~J} / \mathrm{g})$ figure $5 \mathrm{a}$, the second peak can be attributed to the melting, this is confirmed by a thermomicroscopic observation. On cooling, with a rate of $10 \mathrm{~K} / \mathrm{min}$ only one peak is observed. In view to determining the nature of the first endothermic peak an X-ray pattern is performed on the product before the DSC, and an other one after cooling; the two patterns are different, it is possible to deduce that the peak observed at $155^{\circ} \mathrm{C}$ corresponds to a solid-solid phase transition. Moreover, no doubt subsists with a thermomicroscopic observation, the crystals of the low temperature phase $(\alpha)$ have flat form and those of the high temperature $(\beta)$ are long needles. For a DSC performed with a rate of $20 \mathrm{~K} / \mathrm{min}$ the first peak is different, two endotherms are observed and they are followed by an exothermic peak (figure 5b). The peak corresponding to the fusion is observed at $189.7^{\circ} \mathrm{C}$ with an endothermic effect of $107 \mathrm{~J} / \mathrm{g}$ the difference is not really significant but may be explained easily. Once again the thermomicroscopic observation has been of prime importance to explain the phenomenon : at $155^{\circ} \mathrm{C}$ flat crystals are transformed into needles, the difference of shape between the two crystals make the observation easier, the solid-solid transformation is rather slow, and if the heating rate is too fast, all the crystals have not enough time to be transformed, and those of the low temperature form which are not transformed are in a metastable state, and when the temperature of the sample reaches the temperature of the metastable fusion, the low temperature form melts, and then it is the liquid 
which is in a metastable state, if a needle is in contact with a drop of liquid, the liquid will immediately crystallise in the high temperature form. But it is possible to observe that some drop of liquid do not crystallise, if no needles is in contact with the liquid, the germination is more hazardous. This explains the reason why the enthalpy of fusion measured is lower in this case.

At this stage it was not possible to affirm that the transformation was an enantiotropic case, because the transformation of the high temperature form to the low temperature form was not observed on cooling.

A series of experiments was performed with different rate of cooling, the transformation was observed for cooling rate equal or lower than $0.2 \mathrm{~K} / \mathrm{min}$, the temperature of transition was measured at $118^{\circ} \mathrm{C}$. It is important to take in account the kinetic aspect of the transitions, in general no delay is observed for transition when a product is heated, and fusion occurs always at the same temperature (for the same pressure) but solidification appears nearly always with a delay. It has been observed [4] that the delay increases when the cooling rate increases, furthermore for a solidification, to avoid any delay, low cooling rates and an agitation are recommended, but it is not always easily realisable. For solid-solid transition during cooling agitation is useless, only low cooling rates are the solution in view to reaching the equilibrium.

To know if a solid-solid transition is enantiotropic or monotropic a low heating rate in a first approach seems to be the good solution, but using a fast heating rate makes it possible to obtain a metastable equilibrium.

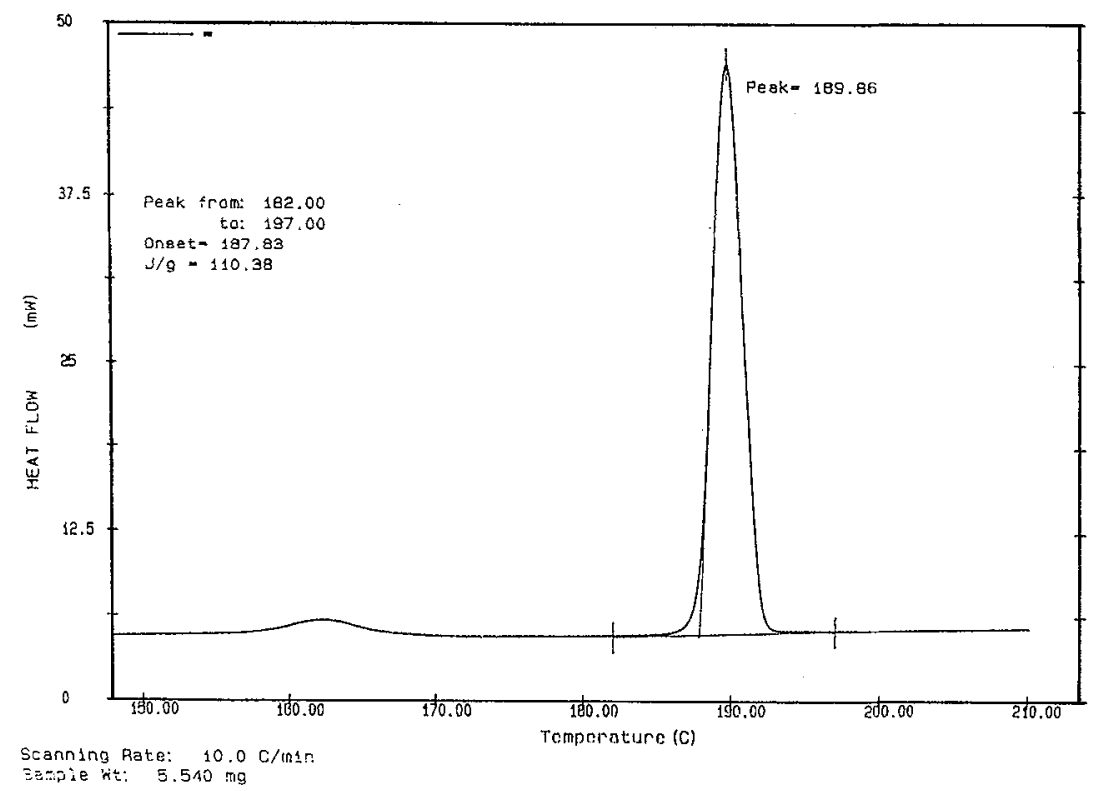

Figure 5a : DSC curve of carbamazepine at $10 \mathrm{~K} / \mathrm{min}$ 


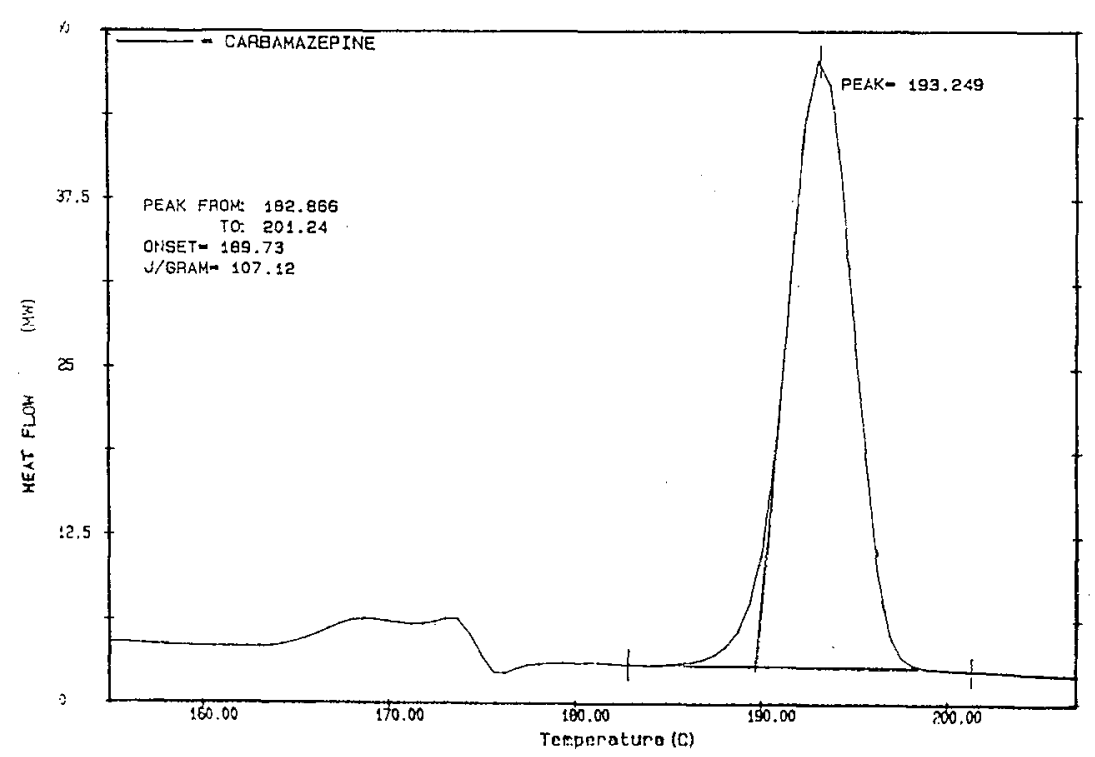

Figure $5 \mathrm{~b}$ : DSC curve of carbamazepine at $20 \mathrm{~K} / \mathrm{min}$

\subsection{The monotropic case}

In this case the metastable phase exists only at high pressure (figure 6). In order to avoid confusion with an enantiotropic case, when only two forms exist we suggest using the symbol $\alpha$ for the stable phase and $\gamma$ for the metastable phase (for $P=1$ bar). The melting temperature of the stable phase $(\alpha)$ is always higher than the metastable phase $(\gamma)$, about the enthalpy of fusion it is not possible to predict which one is the greater between the stable and the metastable fusion, except if the $\mathrm{Cp}$ of the three phases, the liquid and the two solid phases are known.

If the form obtained is the metastable phase, after melting and cooling only the stable phase should crystallise, then on a second run the melting temperature is higher and corresponds to the stable phase, but we shall see later that in some cases things are a bit more complicated. 


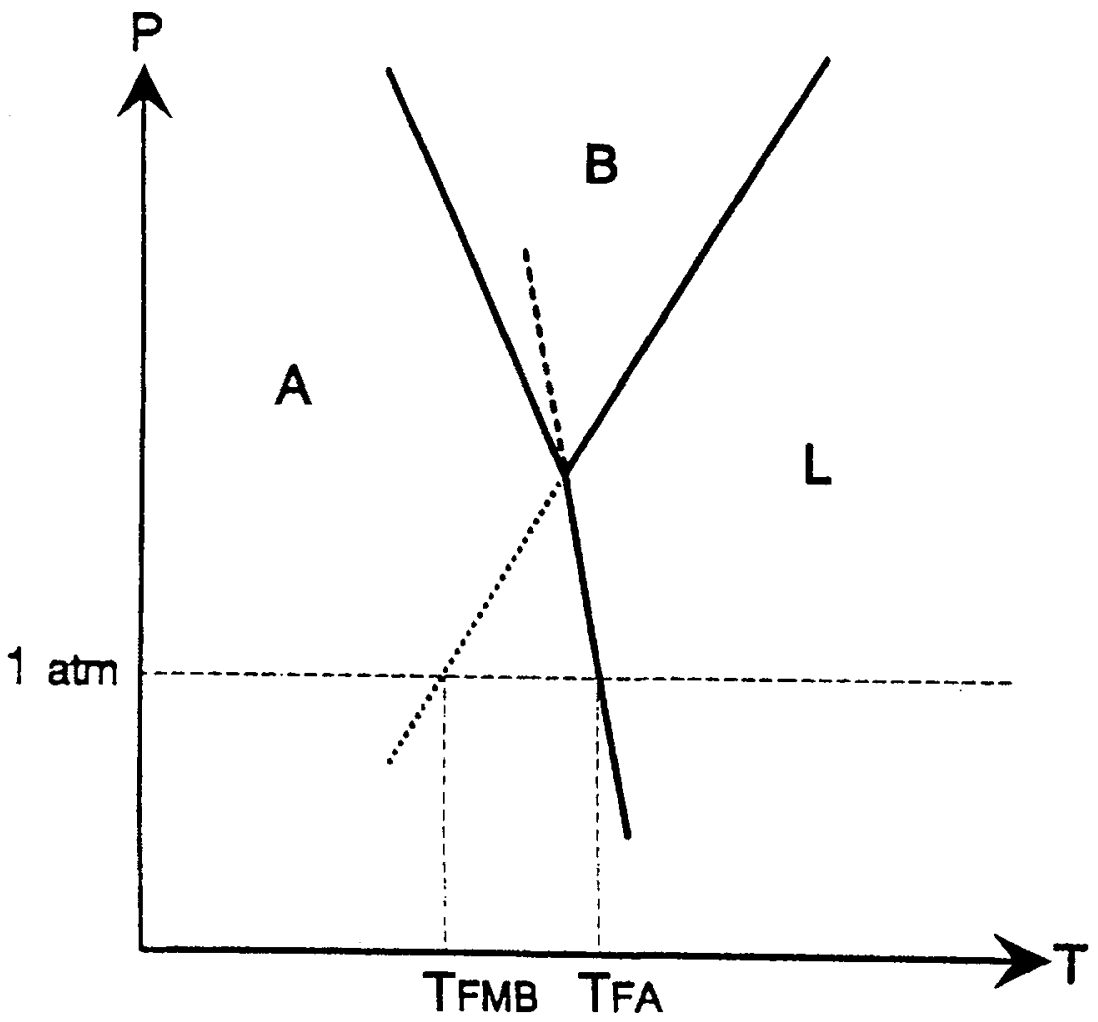

Figure $6: \mathrm{P}, \mathrm{T}$ Unary diagram of a monotropic case

In this paper we present the results for indomethacin, the form obtained depends of the nature of the solvent used for the crystallisation, and in some cases the two forms are present. For crystallisation in water the stable form is obtained, but in methanol and ethanol the metastable phase appears.

With acetone and dichloromethane it is a bit more complicated because solvates are formed.

The melting temperature for $\alpha$ is $\mathrm{T}_{\text {fus }}=159^{\circ} \mathrm{C} \pm 0.3$ with an enthalpy of fusion $\Delta_{\text {fus }} H=$ $109 \mathrm{~J} / \mathrm{g} \pm 0.5$, for the metastable phase $\gamma$, the melting temperature is $\mathrm{T}_{\text {fus }}=151^{\circ} \mathrm{C} \pm 0.3$ with an enthalpy of fusion $\Delta_{\text {fus }} H=96 \mathrm{~J} / \mathrm{g} \pm 0.5$. 
The DSC curve presented in the figure 7, is obtained for a product containing the two forms with a heating rate of $20^{\circ} \mathrm{C} / \mathrm{min}$, it is possible to deduce the percentage of each form by using the values of the enthalpies of melting:

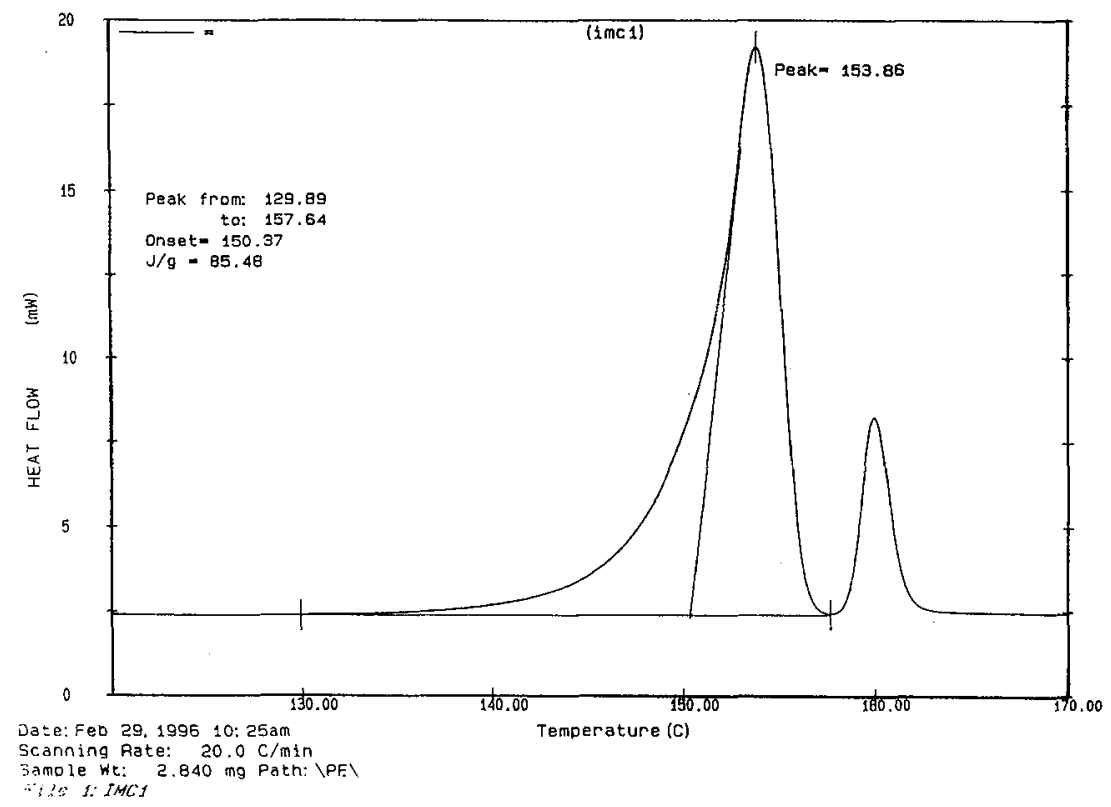

Figure 7 : DSC curve of a mixture of stable and metastable phases of indomethacin

Results are presented in the table 1

\begin{tabular}{|l|l|l|l|}
\hline Pure produce & $\mathrm{T}_{\text {fus }}{ }^{\circ} \mathrm{C}$ & $\Delta_{\text {fus }} H \mathrm{~J} / \mathrm{g}$ & Percentage \\
\hline Form $\gamma$ & 151.0 & 96.3 & $100 \%$ \\
\hline Form $\alpha$ & 159.2 & 108.7 & $100 \%$ \\
\hline Mixing & & & \\
\hline Form $\gamma$ & 150.4 & 85.5 & $89 \%$ \\
\hline Form $\alpha$ & 158.7 & 11.1 & $11 \%$ \\
\hline
\end{tabular}

As we can see the sum of percentage of the two forms is close to $100 \%$, the choice of the heating rate and the amount of product are of a prime importance, the two peaks must not overlap, and the metastable phase $\gamma$ should not crystallise in $\alpha$ after melting. In these conditions this method can be used to follow the kinetic of transformation of the metastable phase in the stable phase versus time. 


\subsection{Non equilibrium behaviour}

3.3.1 Appearance of a metastable phase during cooling.

A recent study on the polymorphism of the $\Delta 2-(2$ Benzofuryl) $\Delta-2$ imidazoline [4], has shown that this compound presents monotropic behaviour, the stable phase $(\alpha)$ melts at $148.5^{\circ} \mathrm{C} \pm 0.5$, with an enthalpy of fusion $\Delta_{\text {furs }} H=153.2 \pm 1 \mathrm{~J} / \mathrm{g}$, after rapid cooling, the metastable phase $(\gamma)$ crystallises, its melting temperature is $\mathrm{T}_{\text {fus }}=138.3^{\circ} \mathrm{C} \pm 0.5$ and the enthalpy of fusion is : $\Delta_{\mathrm{fus}} H=136.6 \pm 1 \mathrm{~J} / \mathrm{g}$. But if the same sample is analysed again a few days later, the melting temperature is $148.5^{\circ} \mathrm{C}$ corresponding to the stable phase. It has been shown in this study that the temperature of crystallisation during cooling depended on the cooling rate; for a weak cooling rate of $0.5^{\circ} \mathrm{C} / \mathrm{min}$ the temperature of solidification was $102^{\circ} \mathrm{C}$ and the solid phase was $\alpha$ (the stable phase), for a cooling rate of $80^{\circ} \mathrm{C} / \mathrm{min}$ the temperature of solidification was $66^{\circ} \mathrm{C}$ and it was the $\gamma$ phase. For cooling rates of 20 or 40 ${ }^{\circ} \mathrm{C} / \mathrm{min}$ the two forms are present. This has been explained by the germination theory [5].

The free energy of nucleation is given by the following formula:

$$
\Delta_{\mathrm{f}} \mathrm{G}=\left(4 \pi \mathrm{r}^{3} / 3\right)\left(\mathrm{G}_{\mathrm{v}}^{\mathrm{s}}-\mathrm{G}_{\mathrm{v}}^{1}\right)+4 \pi \mathrm{r}^{2} \gamma-\Delta_{\mathrm{d}} \mathrm{G}
$$

The first term relative to the volume is negative so we suppose that the solid is a sphere of radius $r, G_{v}^{s}$ and $G_{v}^{\prime}$ are the free energy of the solid and the liquid phase per unit of volume. We are able to evaluate the difference of free energy per mole, an approximation is possible if we consider that the difference between the volumes of the liquid and the solid phases is negligible. The second term relative to the surface is positive, $\gamma$ is the interfacial energy per unity of surface. The third term is necessary when the germination is the result of a solid-solid transition and is due to the elastic distortion. This formula is valuable only in the case of an homogeneous nucleation. In the case of an heterogeneous nucleation it is necessary to take into account the angle of the tangency of the liquid with the solid surface, the formula is multiplied by $S(\theta)$ with:

$$
S(\theta)=(2+\cos \theta)(1-\cos \theta)^{2} / 4
$$

In all the cases the variation of free energy for an heterogeneous nucleation is always lower than in homogeneous case.

The maximum for the free energy of formation is given for $r=r^{*}$, this value is deduced from the derivation of equation (2).

$$
r^{*}=-(2 \gamma) /\left(G^{s}{ }_{v}-G_{v}^{l}\right)
$$

For this value of $r, \Delta G^{*}$ is the free energy of activation of the reaction Liquid $\rightarrow$ Solid

$$
\Delta \mathrm{G}^{*}=\left(16 \pi \gamma^{3}\right) / 3\left(\mathrm{G}_{\mathrm{v}}^{\mathrm{s}}-\mathrm{G}_{\mathrm{v}}^{\mathrm{l}}\right)^{2}
$$


In fact there is competition between the two reactions, Liquid $\Leftrightarrow \alpha$ and Liquid $\Leftrightarrow \gamma$, the reaction which occurs is the one with the lowest energy of activation, and the energy of activation depends of the difference of free energy between the liquid phase and the solid phase at the temperature of solidification.

If the Cp of the liquid and of the two solid phases are not known, it is possible in a first approximation, to use the following formula :

$$
\Delta \mathrm{G}^{(1-s)}=\Delta_{\text {fus }} H\left(1-\mathrm{T} / \mathrm{T}_{\text {fus }}\right)
$$

When the difference between the temperature of fusion and the temperature of solidification increases, the difference between the free energy of activation of the two reactions tends to zero. But with these simplified formula it is not possible to demonstrate the existence of an inversion of values of the free enthalpies of activation. For a complete calculation, the knowledge of the $\mathrm{Cp}$ and the molar volume of each phase versus of the temperature would be necessary. More of this if the reaction which occurs is : Liquid $\Leftrightarrow \gamma$, as $\gamma$ is a metastable phase, this reaction must be considered as a first step, the reaction $\gamma \Leftrightarrow \alpha$ is the second step, an approach of the calculation of the free energy of activation shows that this energy is in the order of range of ten times the value of the free energy of the reaction Liquid $\Leftrightarrow \gamma$, it explains why two or three days later a solid- solid transformation occurs, evidently this kind of reaction is always slow, compared to liquid- solid reactions.

\subsection{Appearance of a metastable phase during heating.}

Another case is the polymorphic transition of the progesterone, actually three forms have been identified, and probably a fourth one (IV), but this last one no X-ray data are available. A recent work performed in our laboratory [6], is in agreement with Cameroni [7,8], The temperatures, the enthalpies and the entropy of fusion for each form are presented in the table 2 below.

\begin{tabular}{|l|l|l|l|l|}
\hline & $\mathrm{T}_{\text {fus }}\left({ }^{\circ} \mathrm{C}\right)$ & $\mathrm{T}_{\text {fus }}(\mathrm{K})$ & $\Delta_{\text {fus }} \mathrm{H}(\mathrm{J} / \mathrm{g})$ & $\Delta_{\text {fus }} \mathrm{S}(\mathrm{J} / \mathrm{g} . \mathrm{K})$ \\
\hline Form I & $129.2 \pm 0.5$ & $402.3_{5} \pm 0.5$ & $83.2 \pm 1$ & $.2068 \pm 0.013$ \\
\hline Form II & $122.2_{5} \pm 0.5$ & $395.4 \pm 0.5$ & $77.8 \pm 1$ & $.1967 \pm .015$ \\
\hline Form III & $108.1 \pm 05$ & $381.7_{5} \pm 05$ & $64.5 \pm 1$ & $.1689 \pm .02$ \\
\hline Form IV & $104.0 \pm 0.5$ & $377.0 \pm 0.5$ & $51.3 \pm 1$ & $.1362 \pm .020$ \\
\hline
\end{tabular}

This case is slightly different from the former one, form $I$ is the stable form, after melting, no crystallisation is observed during cooling, and a glass is formed, when the sample is heated for the second time, a Tg is observed at around $30^{\circ} \mathrm{C}$ and the crystallisation appears, in a lot of cases the exothermic peak is double and sometimes the form I and II are observed, sometimes it is the form II and III, but it has been possible to obtain the forms II and III alone, but the form IV has never been isolated.

The following experiment has been realised, the form I of progesterone is heated in a DSC 7 (Perkin-Elmer), the advantage with this type of apparatus is the low inertia of the 
furnaces, and the possibility to control fast heating and cooling rates (up to $200{ }^{\circ} \mathrm{C} / \mathrm{min}$ ). The first heating is performed with an heating rate of $20^{\circ} \mathrm{C} / \mathrm{min}$ an endothermic peak corresponding to the melting of the form $\mathrm{I}$ is observed for a temperature around $130^{\circ} \mathrm{C}$, during the cooling with a rate of $20^{\circ} \mathrm{C} /$ min no crystallisation is observed, during the second run of heating at $20^{\circ} \mathrm{C} / \mathrm{min}$ an exothermic peak corresponding to a crystallisation is observed, then the fusion occurs at around $122^{\circ} \mathrm{C}$, this has been observed by different authors $[7,8]$, but never explained.

A series of experiments have been performed, with various cooling rates from $200^{\circ} \mathrm{C} / \mathrm{min}$ to $5^{\circ} \mathrm{C} / \mathrm{min}$, but always with the same heating rate of $20^{\circ} \mathrm{C} / \mathrm{min}$; for a cooling rate of $-200^{\circ} \mathrm{C} / \mathrm{min}$ crystallisation occurs at $65^{\circ} \mathrm{C}$ with an enthalpy of $-48.6 \mathrm{~J} / \mathrm{g}$, then only one endothermic peak is observed at $108.5^{\circ} \mathrm{C}$ with an enthalpy of $64.5 \mathrm{~J} / \mathrm{g}$. If the cooling rate is of $150^{\circ} \mathrm{C} / \mathrm{min}$ during the heating the peak of crystallisation observed is double and two peaks of fusion are observed

This could seem to be confusing, but it means that when the undercooled liquid is heated, there is a competition between four reactions, the conditions of germination depend of different parameters, especially if the volume of the sample is small, the nucleation cannot be considered as fully homogeneous and the modelisation is hazardous, the free energy of activation of the tree reactions can be inverted. In fact there is not really a difference between the two cases, and the formation of glass does not modify the conditions of nucleation.

It means that it is not possible to study the polymorhism of a substance without taking in account the kinetic conditions. But it is also interesting to deduce from this study that for monotropic cases a full study for a constant pressure requires to perform DSC at different scanning rate, for heating and cooling, this is a good way to detect the existence of metastable phases by measurement of there melting temperature and there enthalpies of fusion, it is then possible to deduce the percentage of each phase. It is necessary to find the conditions to isolate a metastable phase in order to identify it by X-ray pattern and eventually to isolate single crystals for the resolution of the crystallographic structure.

The best solution is the Calorimetric Analysis versus of the pressure. Currently little number of equipment exits actually, but this will be developed in the future, it is the only way to get a direct access to the unary phase diagram. As it was mentioned above, if the temperature of fusion decreases it means that $\mathrm{dP} / \mathrm{dT}<0$ and we are able to predict that a high pressure form exists, it is a useful inquiry and in this case it is necessary to pay attention to the physical analysis performed for controls. When more than two solids polymorphs exist the resolution without experiments at different pressure is hazardous and speculative.

\section{SOLVATES}

\subsection{Generality}

The study of the polymorphism involves the research of eventual solvates. As a control test, before performing different scanning calorimetry, it is judicious to control the produce obtain from crystallisation in a solvent by using thermogravimetric analysis (TGA).

In most of the cases, if in the crystallographic network of the molecule, cages are formed, it will be possible to include molecules of solvent in the cages of the network, the bonds between the molecule of solvent and the host are weak, and when the temperature 
increases, the volume of the network expands, and the bonds between the atoms of the host molecules increase, then it is possible that the molecules of the solvent escape from the host molecule. The measure of the weight loss versus of the temperature enable us to measure the range of temperature when this happens. Then it is necessary to identify the solvent. The knowledge of the chemical history of the solvate is always useful, because it makes it possible to limit the field of investigations. For a compound with the formula : $\mathrm{AxByCz}, \mathrm{nH}_{2} \mathrm{O}$, when the compound is heated, the following reaction is observed:

$$
\mathrm{AxByCz}, \mathrm{nH}_{2} \mathrm{O} \Leftrightarrow \mathrm{AxByCz}+\mathrm{nH}_{2} \mathrm{O}
$$

If all the molecules of solvent leave the crystal structure at the same time, for one molecule of solvate $\mathrm{n}$ molecules of solvent are released. There is a relation of proportion between them For an initial mass $m$ of solvate, the loss weight measured is $\Delta \mathrm{m}$, the molar mass of the non solvated produce is $\mathrm{M}$, and $\gamma=\mathrm{nM}_{\mathrm{s}} \mathrm{n}$ is the number of mole of solvent for one mole of solvate and $\mathrm{Ms}$ is the molar mass of the solvent.

The determination of $\gamma$ is possible with the following formula:

$$
\Delta \mathrm{m} / \mathrm{m}=\gamma /(\mathrm{M}+\gamma)
$$

In simple case when $\mathrm{n}=1$ it is easy to deduce $M_{s}$. If any doubt exists, a detection of the gas is necessary, different kinds of detectors may be installed at the exit of the TGA gas, pH-meter, IR, CPG or mass spectroscopy.

Below are two examples:

\subsection{Sulfaguanidine}

The formula is $\mathrm{C}_{7} \mathrm{H}_{110} \mathrm{~N}_{4} \mathrm{O}_{2} \mathrm{~S}$ and this compound crystallise with one molecule of water, the curve $m=f(T)$ is presented in figure 8 , for the studied produce, $n=.98$, this value is the average

of ten measurements and the $\sigma=.2$, it means that for this sample the purity is of $98 \mathrm{~mol} \%$. This is confirmed by a DSC measurement realised on the desolvated produce obtained after TGA, the results are treated with the purity program used in DSC.

This example is of a prime interest because TGA can be used as a method of determination of purity for the solvates. 


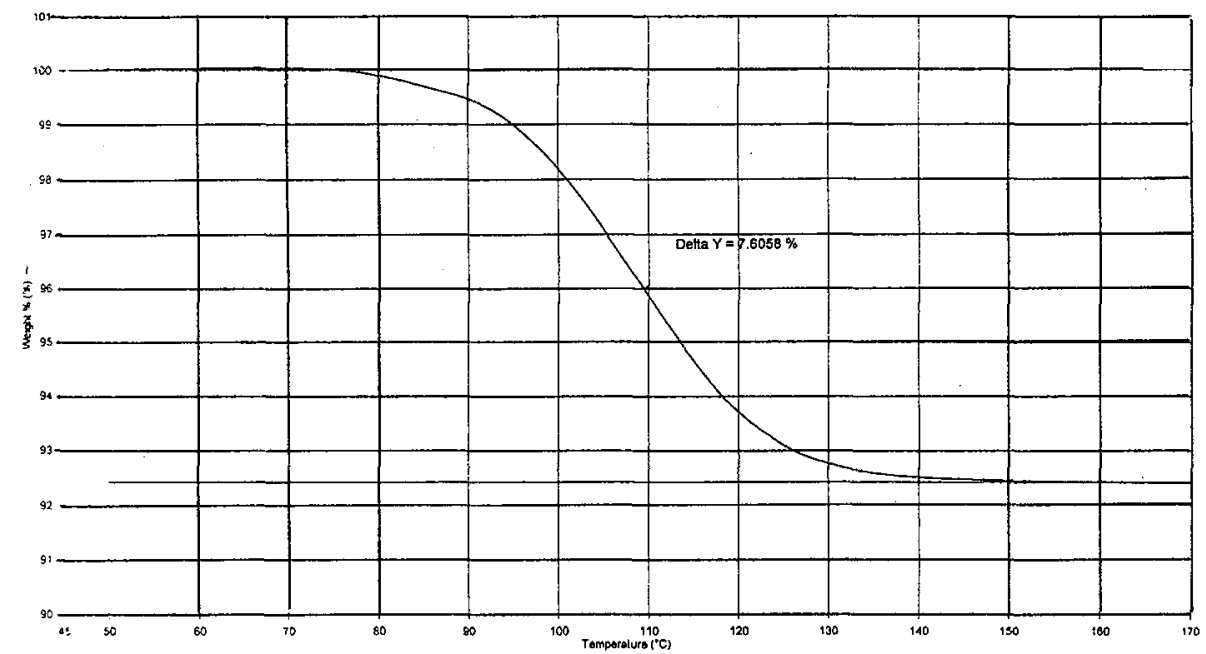

Figure 8 : TGA curve of sulfaguanidine monohydrate

\subsection{Solvates of Indomethacin}

When indomethacin is dissolved in acetone, the X-ray pattern obtained is different from the $\alpha$ and the $\gamma$ form. The DSC and TGA curves are presented on figures $9 \mathrm{a}$ and $9 \mathrm{~b}$. The TGA curve shows that there is no weight loss before $60^{\circ} \mathrm{C}$, if acetone was only adsorbed at the surface of the crystals of indomethacin the evaporation would have started at a lower temperature. The weight loss recorded is of $3.97 \%$ assuming that it corresponds to acetone the value of $\gamma\left(\mathrm{nM}_{\mathrm{s}}\right)$ is 14.78 , as the molecular weight of acetone is $58.08 \mathrm{~g} / \mathrm{mol}$, the solvate is composed of four molecules of indomethacin for one molecule of acetone. In order to identify formally the solvent the gas emitted has been analysed by FTIR, and acetone has been identified. 


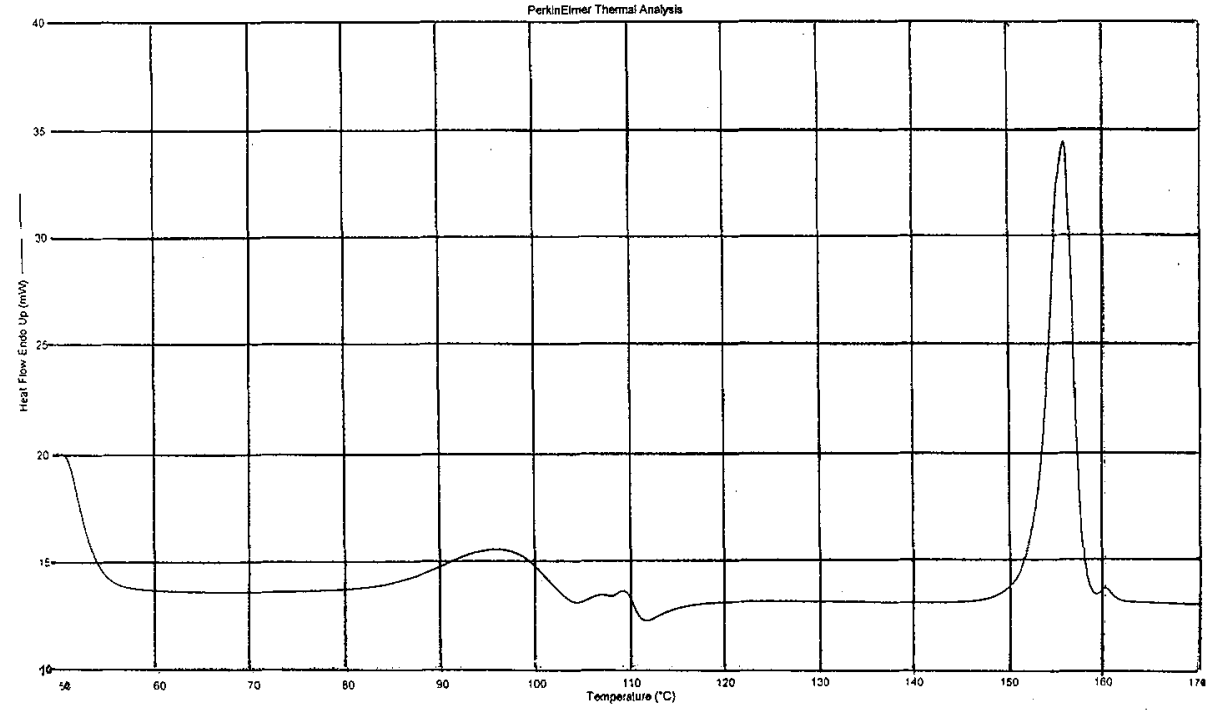

Figure 9a : DSC curve of indomethacin crystallised in acetone

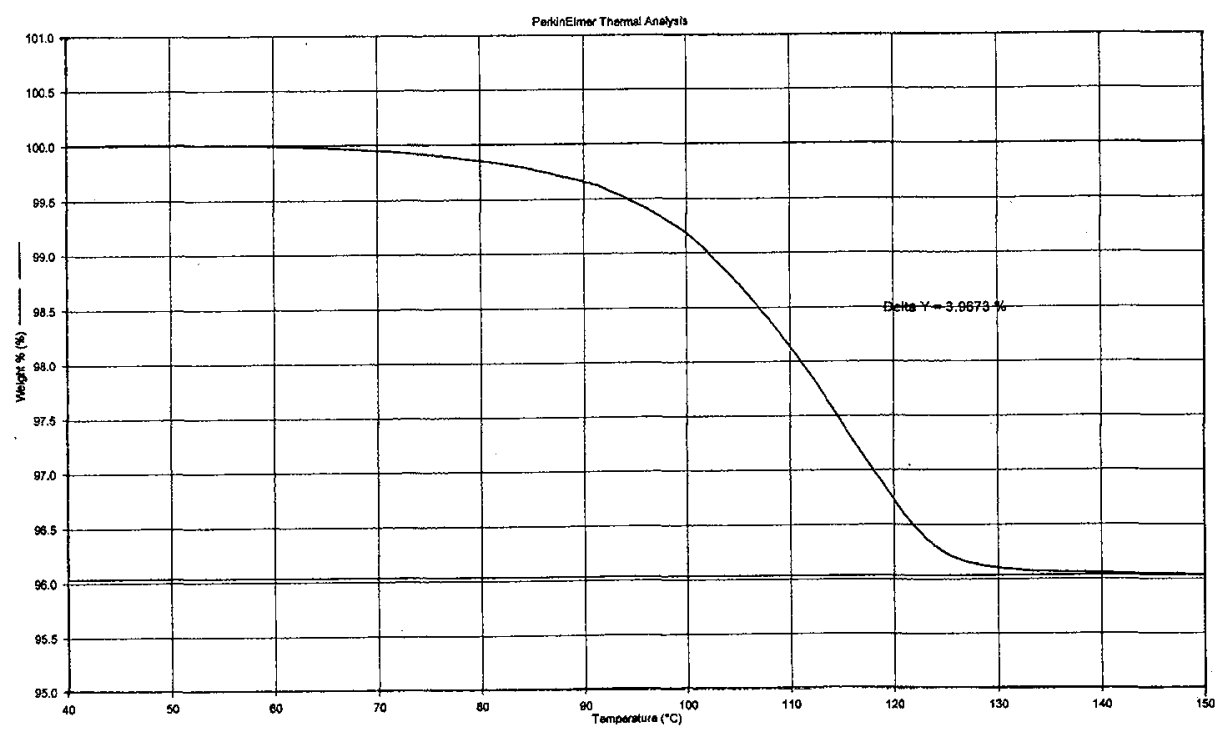

Figure $9 b$ : TGA curve of indomethacin crystallised in acetone 


\subsection{Conclusions}

The ideal solution to study the possible existence of one or several polymorphic forms is the study of the unary phase diagram $P=f(T)$. But more simply, with preparation in different solvents it is possible to reveal metastable phases which may be characterised by Xray. Different scanning calorimetry performed with various heating rates, even in non equilibrium conditions is indispensable for the understanding of the thermodynamic behaviour. Thermogravimetric analysis is absolutely necessary in view to detect the presence of solvates or on the contrary to be sure that no solvates are present, this method can be completed by IR or mass detection. Polymorph studies are of undeniable importance for pharmaceutical industry in view to obtaining the marketing authorisation for drugs.

\section{Bibliography}

[1] J.C. Mathieu, F. Durand, E. Bonnier. J. Chem, Phys.11-12, (1965), 1289-

[2] C.H.P. Lupis, J.F. Elliot. Acta. Met. 15, (1967) 265-

[3]) J.K. Halebian, R.T. Koda, J.A. Biles. J. Pharm. Sci. 60, (10), (1971) 1488-1491.

[4] B. Legendre, G. Baziard-Mouysset, M. Anastassiadou, J.M. Leger, M. Payard.

Journal of thermal analysis and calorimetry, in press.

[5] R.Beker, W. Doring, Ann Physik, 24, (193 5), 719.

[6]) B. Legendre, Y. Feutelais. "Thermodynamic study of Indomethacin"

To be published.

[7] R.Cameroni, G.Gamberini, M.T. Bernabei, M. Facchini. Il Farmaco. Ed.Prat., 28,(12), (1973) 621-635

[8] R.Cameroni, G.Gamberini, M.T. Bernabei. Il Farmaco. Ed.Prat., 28,(12), (1973) 636-642

\section{Figure caption}

Figure 1a : P,T diagram of water

Figure $1 \mathrm{~b}: \mathrm{P}, \mathrm{T}$ diagram of water at high pressure

Figure $2: P, T$ Unary phase diagram of an enantiotropic case

Figure 3 : Heat content versus of $\mathrm{T}, \Delta_{298}^{\mathrm{T}} H=\mathrm{f}(\mathrm{T})$.

Figure 4 : Free enthalpy versus of $\mathrm{T}, G=\mathrm{f}(\mathrm{T})$

Figure 5a : DSC curve of carbamazepine at $10 \mathrm{~K} / \mathrm{min}$

Figure $5 b:$ DSC curve of carbamazepine at $20 \mathrm{~K} / \mathrm{min}$

Figure $6:$ P,T Unary diagram of a monotropic case

Figure 7 : DSC curve of a mixture of stable and metastable phases of Indomethacin

Figure 8 : TGA curve of Sulfaguanidine monohydrate.

Figure 9a : DSC curve of indomethacin crystallised in acetone

Figure $9 b$ : TGA curve of indomethacin crystallised in acetone 
Annexe

\section{DISSOLUTION}

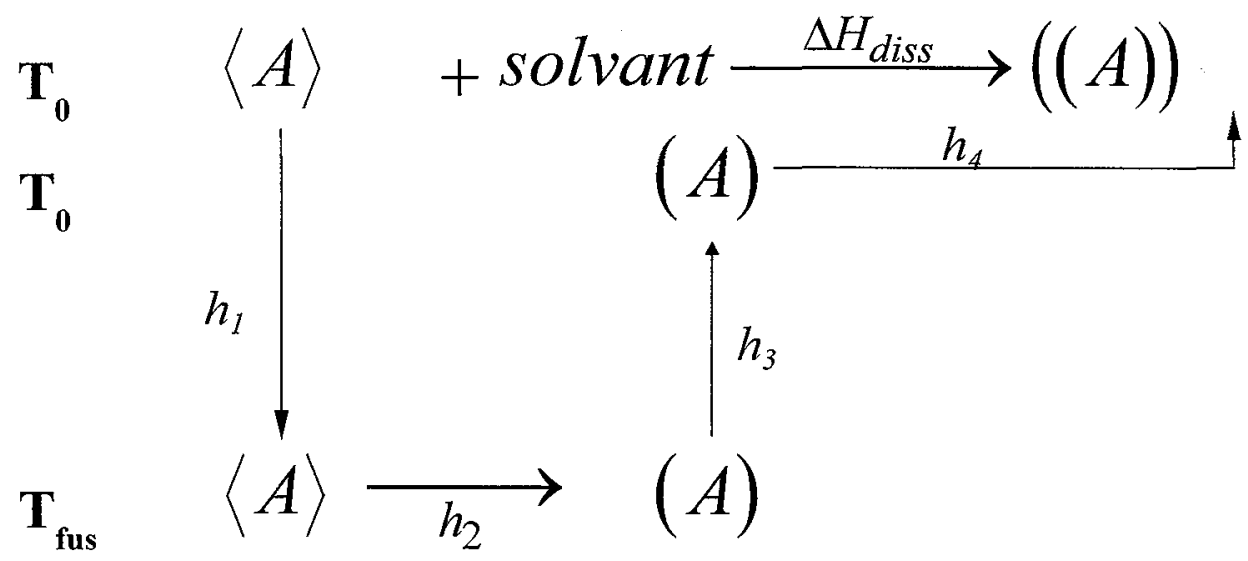

$$
\Delta H_{\text {diss }}=\mathbf{h}_{1}+\mathbf{h}_{2}+\mathbf{h}_{3}+\mathbf{h}_{4}
$$

$$
\mathrm{h}_{\mathrm{l}}=\int_{T_{0}}^{T_{\text {fus }}} c_{p}^{s} d T \quad \quad \mathbf{h}_{2}=\Delta \boldsymbol{H}_{\mathrm{fus}} \text { (enthalpy of fusion) }
$$$$
\int_{\mathbf{h}_{3}=}^{T_{0}} \int_{T_{f u s}}^{l i q} d T \quad \mathbf{h}_{4}=\Delta \boldsymbol{H}_{\text {mix }} \text { (enthalpy of mixing of the two iquids) }
$$$$
\Delta \boldsymbol{H}_{\text {diss }}=\int_{T_{0}}^{T_{f u s}}\left(c_{p}^{s}-c_{p}^{l i q}\right) d T_{+\Delta H_{\text {fus }}+\Delta H_{\text {mix }}}
$$

$\Delta H_{\text {fus }}$ always positive $\Delta H_{\text {mix }}$ positive or negative

$h_{1}+h_{3}$ positive but close to zero

$$
\mathrm{V}_{\text {diss }}=\mathrm{f}\left(\Delta H_{\text {fus }}\right)
$$

